\title{
The effect of mental fatigue on half-marathon performance: a pragmatic trial
}

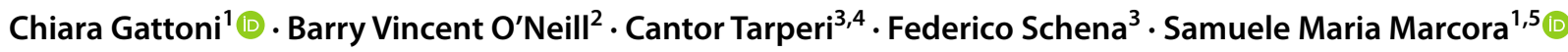

Received: 11 February 2021 / Accepted: 28 May 2021 / Published online: 24 June 2021

(c) The Author(s) 2021

\begin{abstract}
Purpose It is well established that mental fatigue impairs performance during lab-based endurance tests lasting less than $45 \mathrm{~min}$. However, the effects of mental fatigue on longer duration endurance events and in field settings are unknown. The aim of this study was to investigate the effect of mental fatigue on performance during a half-marathon race.

Methods Forty-six male amateur runners (means \pm SD: age $43.8 \pm 8.6$ years, $\dot{V} O_{2 p e a k} 46.0 \pm 4.1 \mathrm{ml} / \mathrm{kg} / \mathrm{min}$ ) completed a half-marathon after being randomly allocated to performing a 50-min mentally fatiguing task (mental fatigue group) or reading magazines for $50 \mathrm{~min}$ (control group). Running speed, heart rate, and perceived effort were measured during the race.

Results Runners in the mental fatigue group completed the half-marathon approximately 4 min slower (106.2 $\pm 12.4 \mathrm{~min})$ than those in the control group (102.4 $\pm 10.2 \mathrm{~min}$ ), but this difference was not statistically significant (Cohen's $d=0.333$; $p=0.265$ ). However, equivalence was not established $[t(40.88)=0.239, p=0.594]$ and equivalence testing analysis excluded a beneficial effect of mental fatigue on half-marathon performance.

Conclusion Due to its posttest-only design and the achievable sample size, the study did not have enough power to provide evidence that the observed 4-min increase in half-marathon time is statistically significant. However, equivalence testing suggests that mental fatigue has no beneficial effect on half-marathon performance in male amateur runners, and a harmful effect cannot be excluded. Overall, it seems prudent for endurance athletes to avoid mentally fatiguing tasks before competitions.
\end{abstract}

Keywords Endurance performance $\cdot$ Perception of effort $\cdot$ Cognitive fatigue $\cdot$ Aerobic exercise $\cdot$ Equivalence testing $\cdot$ Perceived exertion

\section{Introduction}

Mental fatigue is defined as a psychobiological state caused by prolonged cognitive exertion that can impair cognitive performance and/or induce feelings of tiredness and lack of energy [1]. Over the past decade, several experimental

Samuele Maria Marcora

samuele.marcora@unibo.it

1 School of Sport and Exercise Sciences, University of Kent, Chatham Maritime, UK

2 Brain Health Department, Nestlé Research, Société Des Produits Nestlé SA, Lausanne, Switzerland

3 Department of Neurosciences, Biomedicine and Movement Sciences, University of Verona, Verona, Italy

4 Department of Clinical and Biological Sciences, University of Turin, Turin, Italy

5 Department of Biomedical and Neuromotor Sciences, University of Bologna, Bologna, Italy studies have shown that mental fatigue can also impair endurance (aerobic) performance in physically active, healthy adults in normal ambient conditions (i.e. temperature: $18-22{ }^{\circ} \mathrm{C}$; humidity: $45-60 \% \mathrm{RH}$ ) [2-8]. In hot ambient conditions, the effect of mental fatigue on endurance performance is less clear with one study reporting no significant effect [9] and another reporting both separate and combined effects of pre-exercise heat stress and mental fatigue on endurance performance in the heat [10].

In the studies conducted so far, endurance performance was measured with time to exhaustion tests or time trials [11] lasting between 3 and $45 \mathrm{~min}$ [8]. Furthermore, these experiments have been conducted in standardised environments, such as laboratories $[2,3,5-8,12]$ and an indoor track [4]. To the best of our knowledge, there is no published study about the effects of mental fatigue in outdoor settings and during mass-start competitions lasting more than $45 \mathrm{~min}$ (long-term endurance performance). This is not surprising because, due to logistics and testing issues, it is extremely 
difficult to conduct randomised controlled studies during real endurance competitions. Indeed, only a handful of such pragmatic trials have been published [13-18].

Qualitative studies have found that athletes can experience mental fatigue during both training and competition periods [19-22]. In particular, travelling, strict schedules, seasonal variations, repetitive tasks, novel tasks, new environments and experiences, over-analyses of competition, professional commitments as well as an overuse of technology and mobile apps are among the major factors associated with mental fatigue [20, 21, 23, 24]. In addition, the sustained cognitive effort required for self-regulation during prolonged and strenuous endurance competitions may also induce mental fatigue and impair performance [25]. Consequently, investigating whether mental fatigue can negatively affect performance during real endurance competitions lasting more than $45 \mathrm{~min}$ is warranted.

To have a more ecologically valid insight into the effect of mental fatigue on long-term endurance performance, the main aim of the current study was to test the hypothesis that a mentally fatiguing task reduces performance during a subsequent official half-marathon race in amateur runners. The effects of mental fatigue on pacing pattern, heart rate and perception of effort during the race were also investigated.

\section{Methods}

\section{Participants}

Forty-eight male amateur long-distance runners were recruited. Two participants in the experimental treatment were excluded due to injury and premature exhaustion during the race. All participants signed a written, informed consent prior to participation. All procedures used were approved by the Ethics Committee from the University of Verona (approval number: 165038) and were conducted in conformity with the Declaration of Helsinki.

Participants were recruited and tested over three editions (2015, 2016, 2017) of Run4Science (website: https://www. r4s.it/en/home-en/). Run4Science is a competitive event (marathon and half-marathon) organised by the University of Verona (in collaboration with the Italian Athletics Federation and with the patronage of the city council) to give scientists the opportunity to study long-distance running in real-life conditions.

Subjects eligible for this study were involved in regular running (aerobic) training, free of any known disease, injury and medical treatment. To have a homogeneous fitness level, the recruited runners were first asked to complete a trainingcompetition history questionnaire and a physical activity rating scale (PA-R) [26]. Only those runners with a physical activity rating above 6 were included in the experiment.
Participants were not aware of the real aim and hypotheses of the experiment. They were told that the study aimed to investigate the effects of two different kinds of cognitive activities on physiological and psychological responses to a half-marathon race.

\section{Study protocol}

A between-subject, posttest-only, randomized controlled design was used for this pragmatic trial. Participants were randomly asked either to perform a 50-min mentally fatiguing task immediately before the half-marathon (experimental treatment) or to read some magazines for the same amount of time, always prior to the competition (control treatment). To control for the potential confounding effects of atmospheric conditions and other variables that may differ between the three races, participants at each data collection constituted a separate block and were randomly allocated to treatment as follows. To create two groups of equal size and similar peak oxygen consumption $\left(\dot{V} O_{2 p e a k}\right)$ at baseline, random allocation to treatment was performed in blocks of two participants ranked according to their $\dot{V} O_{2 p e a k}$ which was estimated using a validated multiple regression equation that takes into account subjects' PA-R [26], body mass index (BMI), gender and age [27]. Participants' features (general and per group) are reported in Table 1. For statistical analysis, the three blocks of participants (one for each data collection) were pooled together.

The study took place at the Department of Neurosciences, Biomedicine and Movement Sciences, University of Verona. Both psychological questionnaires and cognitive tasks were carried out in a standardised lecture room. The half-marathon race took place in proximity to the Department (See Fig. 1 for the course).

One week prior to the half-marathon, runners were familiarized with the use of the 100-point rating of perceived exertion (RPE) scale [28] and other questionnaires/ scales (see "Psychological questionnaires"). They were also informed to drink around $35 \mathrm{ml}$ of water per $\mathrm{kg}$ of body weight [29], to sleep at least $7 \mathrm{~h}$, to refrain from alcohol consumption and to avoid strenuous exercise within the $24 \mathrm{~h}$ preceding the experiment. Participants were also asked not to consume any caffeine and nicotine for at least $3 \mathrm{~h}$ before the experiment.

On the race day, after a standardised breakfast (07:00-07:30 am), subjects were divided into two groups and asked to sit down either in the front (mental fatigue) or the back (control) of a lecture room. The experiment started at 08:00 am.

Participants were required to complete a mental and a physical fatigue scale (see "Psychological questionnaires"). They were then asked to perform either the mental fatigue or the control task for $50 \mathrm{~min}$ (see "Treatment"). Immediately 
Table 1 Participants features $(N=46)$. Data are shown as means \pm SD

\begin{tabular}{lcccc}
\hline & $\begin{array}{l}\text { Mental fatigue } \\
(N=22)\end{array}$ & $\begin{array}{l}\text { Control } \\
(N=24)\end{array}$ & $\begin{array}{l}\text { Total participants } \\
(N=46)\end{array}$ & Total range \\
\hline Age (years) & $43.1 \pm 10.0$ & $44.4 \pm 7.2$ & $43.8 \pm 8.6$ & $20.3-59.0$ \\
Height $(\mathrm{m})$ & $1.76 \pm 0.07$ & $1.77 \pm 0.06$ & $1.76 \pm 0.06$ & $1.55-1.89$ \\
Weight $(\mathrm{kg})$ & $74.0 \pm 7.2$ & $73.0 \pm 6.4$ & $73.5 \pm 6.7$ & $60.0-87.0$ \\
BMI $\left(\mathrm{kg} / \mathrm{m}^{2}\right)$ & $23.9 \pm 1.8$ & $23.3 \pm 1.6$ & $23.6 \pm 1.7$ & $20.8-27.1$ \\
PA-R & $6.8 \pm 0.5$ & $6.9 \pm 0.3$ & $6.8 \pm 0.4$ & $6-7$ \\
Estimated $\dot{V} O_{2 \text { peak }}(\mathrm{ml} / \mathrm{kg} / \mathrm{min})$ & $45.9 \pm 4.8$ & $46.0 \pm 3.6$ & $46.0 \pm 4.1$ & $35.8-56.2$ \\
Training/Week $(\mathrm{h})$ & $5 \pm 3$ & $5 \pm 3$ & $4 \pm 2$ & $1-15$ \\
Training/Year $($ weeks $)$ & $45 \pm 7$ & $46 \pm 5$ & $45 \pm 6$ & $30-52$ \\
\hline
\end{tabular}

$B M I$, Body Mass Index; $P A-R$, Physical Activity Scale; $\dot{V} O_{2 p e a k}$, peak oxygen uptake

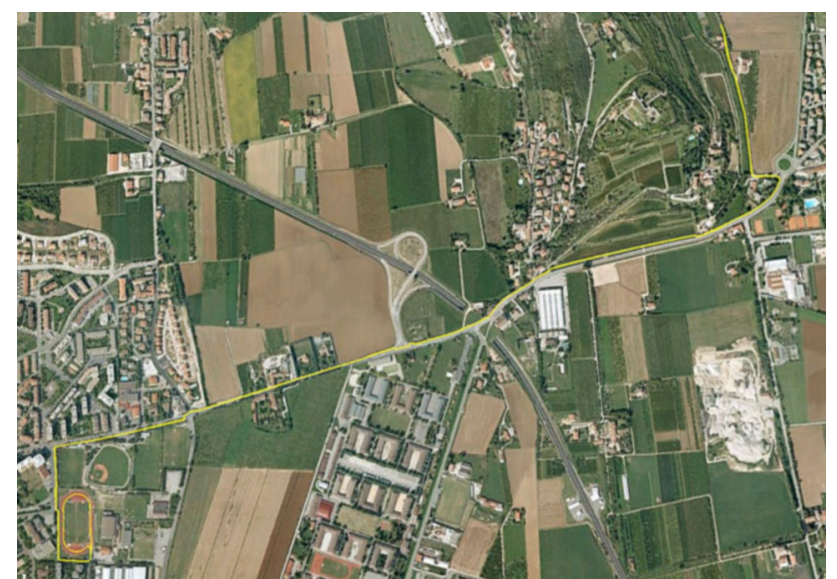

Fig. 1 The 7-km circuit course of the race that was repeated three times

after the task, subjects were required to complete the same fatigue scales completed at baseline. To assess subjective workload perceived during the treatment, and motivation and expectations related to the half-marathon race, participants completed a workload-related multidimensional scale, a motivation questionnaire and an expectation scale, respectively (see "Psychological questionnaires").

After the treatment and the completion of the psychological questionnaires participants performed the half-marathon race (atmospheric conditions: first data collection: temperature: $20.5{ }^{\circ} \mathrm{C}$; humidity: $35 \% \mathrm{RH}$; barometric pressure: $1013 \mathrm{hPA}$; second data collection: temperature: $21.2{ }^{\circ} \mathrm{C}$; humidity: 53\% RH; barometric pressure: $1013 \mathrm{hPA}$; third data collection: temperature: $24.2{ }^{\circ} \mathrm{C}$; humidity: $47 \% \mathrm{RH}$; barometric pressure: $1029 \mathrm{hPA}$ ). The course was a controlled $7-\mathrm{km}$ circuit to be completed three times. The starting point was located inside the University outdoor track. Participants were equipped with a shoe race chip through which lap and overall performance times were taken. At the end of each lap, athletes were required to run on the track and pass through the start, where RPE was collected. RPE was measured using the 100-point scale [28]. Two big posters of the same scale were placed in proximity of the starting point at 50-m distance between each other. Subjects' heart rate (HR) and running speed were continuously measured throughout the entire race using GPS watches (Polar V800, Polar Electro Oy, Kempele, Finland). Participants were asked to do a 10-min warm-up immediately before the race. Participants were free to drink ad libitum during the race.

At the end of the race, participants were asked to go back to the lecture room and to complete the same physical and mental fatigue scales related to the half-marathon race (see "Psychological questionnaires").

\section{Treatment}

In the mental fatigue group, treatment consisted in performing a 50-min mentally fatiguing task on a tablet screen (iPad Mini 2, Apple, California, USA). The mentally fatiguing task was developed by Axon Sports (Phoenix, Arizona, USA), and consisted of five consecutive blocks of 10-min, during which a simple response task and a search response task were run. In the simple response task participants were required to detect and press a visual stimulus (a green target) appearing randomly in the centre of the screen (stimulus frequency between 500 and $1500 \mathrm{~ms}$ ). The total duration of the simple response task was $45 \mathrm{~s}$ within each block. In the search response task participants were asked to detect and press a green target (go stimulus) and not to respond to a red target (no-go stimulus). Both stimuli appeared randomly in different positions on the screen (stimulus frequency between 750 and $1000 \mathrm{~ms}$ ). A bleep sound was elicited in case of incorrect response. The total duration of the search response task was 9 min and $15 \mathrm{~s}$ within each block. In the control group, treatment consisted in reading some magazines for $50 \mathrm{~min}$, as it is considered a relaxing leisure activity [30]. Participants were continuously monitored by the researchers to guarantee compliance with both treatments. 


\section{Psychological questionnaires}

\section{Subjective workload}

The multidimensional rating scale NASA Task Load Index (TLX) [31] was used to estimate subjective workload that participants experienced during the treatment. The NASA TLX includes six subscales which determine workload: mental demand, physical demand, temporal demand, performance, effort and frustration. Subjects were asked to circle one of the 20 equal line intervals present on each of the six subscales at the point which matched their experience. All subscales have two endpoint descriptors, "very low" on the left and "very high" on the right, except for the performance subscale which goes from "good" to "poor". The score of each subscale was multiplied by 5 , obtaining final scores between 0 and 100 .

\section{Mental and physical fatigue}

The same type of scale present in the NASA TLX [31] was used to assess mental fatigue and physical fatigue before and after the treatment. Subjects were asked to circle one of the 20 equal line intervals present on each scale based on their current feelings of mental and physical fatigue. The two scales ends are anchored by descriptors defining the extreme feelings of fatigue: "no fatigued at all" and "extremely fatigued". The score of each scale was multiplied by 5, obtaining final scores between 0 and 100 . Change scores (score after treatment minus score before treatment) were then calculated for both physical and mental fatigue.

\section{Motivation}

Intrinsic motivation and success motivation scales [32] were used to assess motivation related to the half-marathon race. Each scale includes 7 items to be scored on a 5-point Likert scale (where $0=$ not at all, $1=$ a little, $2=$ somewhat, $3=$ very much, $4=$ extremely). The total range of scores for each scale is between 0 and 28 .

\section{Performance expectations}

The same scale used in the NASA TLX [31] was also adopted to measure participants' expectations related to the half-marathon race. Subjects were asked to circle one of the 20 equal line intervals present on the scale based on how well they expected to perform their race. The scale ends are anchored by descriptors defining the extreme expectations: "much better than my personal best" and "much worse than my personal best". A third descriptor "my personal best" was added to the centre of the scale. The score of the scale was multiplied by 5 , obtaining final scores between 0 and 100 .

\section{Statistical analysis}

The Shapiro-Wilk test, histograms, Q-Q plots and boxplots were used to check all data for normality. If data were not normally distributed, non-parametric tests were used.

Independent t-tests were used to analyse between-group differences in the half-marathon time, the motivation and expectation scores, and the change score of mental fatigue. Because the change score of physical fatigue and the workload scores were not normally distributed, non-parametric Mann-Whitney tests were used to analyse between-group differences in these variables.

$2 \times 3$ mixed-model ANOVAs (group $x$ distance) were used to analyse the main effects and interaction of these two factors on running speed, RPE, HR and the ratio between $\mathrm{RPE}$ and running speed during the half-marathon. When the assumption of sphericity was not met, the Greenhouse-Geisser correction was used. Significant interactions were followed up by testing simple main effects of the group at each distance with Bonferroni's correction.

Because of logistical constraints, performing another half-marathon as a pretest was not feasible. Therefore, we had to implement the less statistically powerful betweengroup, posttest-only experimental design. Given the additional constraints in terms of the sample size achievable over three editions of the race, and the small-to-moderate effect size found in our previous studies, we did not expect to have enough power to detect a statistically significant effect on our main outcome variable (half-marathon time) with the traditional null hypothesis significance testing (NHST) approach described above. To provide some useful information to sport scientists, coaches and athletes even in the absence of a statistically significant effect of mental fatigue on half-marathon performance, we originally planned to use the magnitude based inference (MBI) approach proposed by Hopkins and colleagues [33]. However, before we conducted the statistical analysis, the MBI approach had been heavily criticised by statisticians [34] and it is no longer recommended for use in sport science research $[35,36]$. Therefore, we decided to use another statistical approach, called two one-sided tests (TOST) equivalence testing procedure [20, 21]. Statisticians have specifically recommended the use of TOST equivalence testing in addition to NHST [37] to prevent common misinterpretation of $p$ values larger than the alpha level [38] as support for the absence of a true effect $[39,40]$. This analysis adds important information to the NHST as it tests the presence or the absence of meaningful 
effects. In the present study, the TOST procedure was used to test the hypothesis that mental fatigue has no meaningful effect on half-marathon time (equivalence). The smallest effect size of interest (SESOI) was decided a priori, with lower $(\Delta \mathrm{L})$ and upper $(\Delta \mathrm{U})$ bounds set to -0.26 and 0.26 (i.e. -2.947 and $2.947 \mathrm{~min}$ on a raw scale). $\Delta \mathrm{L}$ and $\Delta \mathrm{U}$ corresponded to the latest meta-analysis effect size (Hedge's g) of cognitive exertion on aerobic performance [17].

In the NHST analysis, statistical significance was accepted at $p<0.05$ level. All data are presented as means \pm SD, unless otherwise stated. RStudio (version 1.1.4; RStudio, Boston, MA) was used for the TOST equivalence testing analysis. The SPSS (version 23.0; SPSS, Chicago, IL) statistical package was used for all the other data analyses.

\section{Results}

\section{Manipulation checks}

The difference in the mental fatigue change score was significant (mental fatigue 22.0 \pm 21.6 ; control 7.1 \pm 16.9 ; Cohen's $d=0.773$; $p=0.012$ ), showing a greater increase in the mental fatigue group compared to the control group (Fig. 2). No significant between-group difference was found for the physical fatigue change score (mental fatigue: $M d n=0.00$, Interquartile Range $(I Q R)=3.75$; control: $M d n=0.00$, $I Q R=10.00 ; U=245.00, z=-0.44, r=-0.07 ; p=0.662)$. The multidimensional scale NASA TLX completed immediately after the treatment showed a trend toward significant higher values of mental demand in the mental fatigue group $(M d n=42.50, I Q R=61)$ compared to the control group

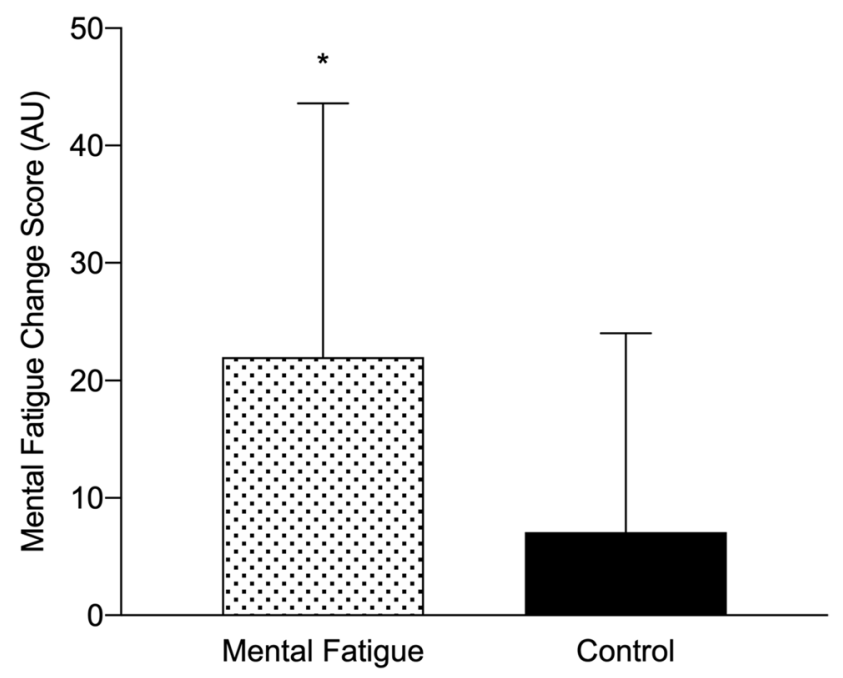

Fig. 2 Effect of mentally fatiguing task on subjective ratings of mental fatigue. AU Arbitrary Units. Data are displayed as change scores mean \pm SD. *Significant effect of the experimental treatment $(p<0.05)$
$(M d n=25.00, I Q R=30)(U=176.50, z=-1.93, r=-0.30$; $p=0.053)$. The same multidimensional scale also revealed that the mental fatigue group $(M d n=47.50, I Q R=53)$ provided significantly higher ratings of effort $(M d n=25.00$, $I Q R=45)(U=164.00, z=-2.21, r=-0.33 ; p=0.027)$ and temporal demand (mental fatigue: $M d n=50.00, I Q R=53$; control: $M d n=10.00, I Q R=14 ; U=106.00, z=-3.49, r=$ $-0.51 ; p<0.001)$ than the control group. Ratings of physical demand, performance and frustration were not significantly different between the two groups.

Intrinsic motivation (mental fatigue $23.0 \pm 2.5$; control $23.1 \pm 3.0$; Cohen's $d=0.030 ; p=0.919$ ) and success motivation (mental fatigue $16.4 \pm 3.1$; control $16.1 \pm 3.3$; Cohen's $d=0.074 ; p=0.802$ ) did not differ significantly between the two groups. Expectations related to the half-marathon performance also did not differ significantly between groups (mental fatigue 43.2 \pm 22.4 ; control 45.2 \pm 14.1 ; Cohen's $d=0.110 ; p=0.713)$.

\section{Effect of mental fatigue on half-marathon performance}

The NHST analysis (independent t-test) showed no significant difference between the two groups (mental fatigue $106.2 \pm 12.4 \mathrm{~min}$; control $102.4 \pm 10.2 \mathrm{~min} ; 95 \% \mathrm{CI}$ [ $-3.0,10.5 \mathrm{~min}]$; Cohen's $d=0.333 ; p=0.265$ ) (Fig. 3). The TOST procedure revealed no significant equivalence $(t(40.88)=0.239, p=0.594)$. Inspection of the $90 \%$ confidence limit of the difference between the two groups $(90 \%$ CI $[-1.9,9.4 \mathrm{~min}])$ in relation to the upper and lower equivalence bounds of the SESOI (Fig. 4) shows that whilst the presence of a beneficial effect of mental fatigue can be excluded, the presence of a harmful effect cannot be excluded. Indeed, whereas the test against $\Delta_{L}$ shows

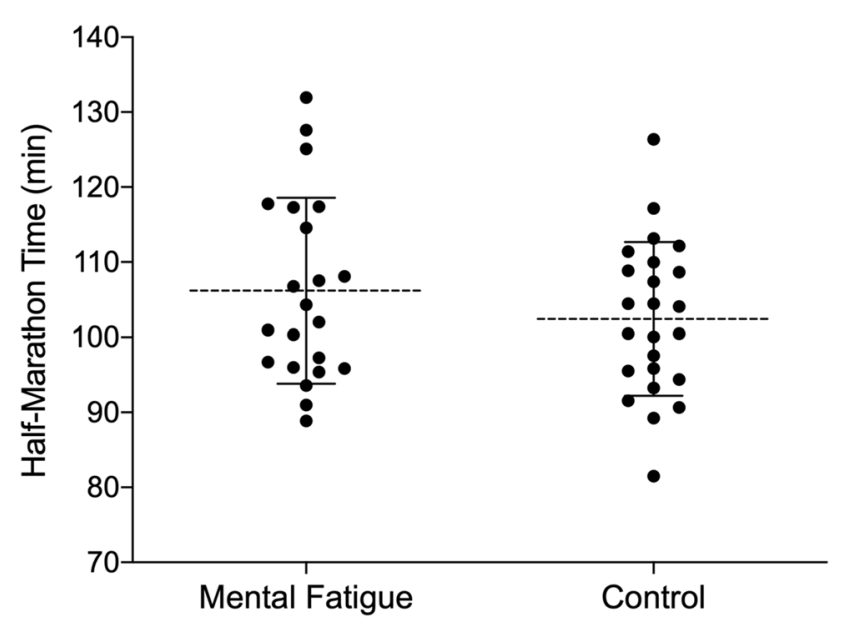

Fig. 3 Half-marathon time in the mental fatigue group and the control group. Dots represent individual data whilst horizontal lines represent means (open lines) and standard deviations (solid lines) 
that differences equal or smaller than -2.947 min can be rejected, the test against $\Delta_{U}$ indicates that effects at least as extreme as 2.947 min cannot be rejected.

\section{Effects of mental fatigue on running speed, HR, and RPE}

No significant group $\mathrm{x}$ distance interactions were found on running speed $(p=0.910), \operatorname{RPE}(p=0.582), \operatorname{HR}(p=0.829)$ and RPE/running speed $(p=0.530)$. Running speed showed a significant decrease over distance in both groups $(p<0.001)$. However, no significant difference was found between groups (Cohen's $d=0.274, p=0.358$ ) (Fig. 5a). RPE increased significantly over distance in both groups $(p<0.001)$. However, no significant difference was found between groups (Cohen's $d=0.162, p=0.586$ ) (Fig. 5b). HR showed a significant increase over distance in both groups $(p<0.001)$. However, no significant difference was found between groups (Cohen's $d=0.391, p=0.192$ ) (Fig. 5c). RPE/running speed also increased significantly over distance in both groups $(p<0.001)$. However, no significant difference was found between groups (Cohen's $d=0.260$, $p=0.422)$ (Fig. 5d).

\section{Discussion}

\section{Effect of mental fatigue on half-marathon performance}

Participants reported that the mentally fatiguing task was mentally demanding and effortful, and it increased the

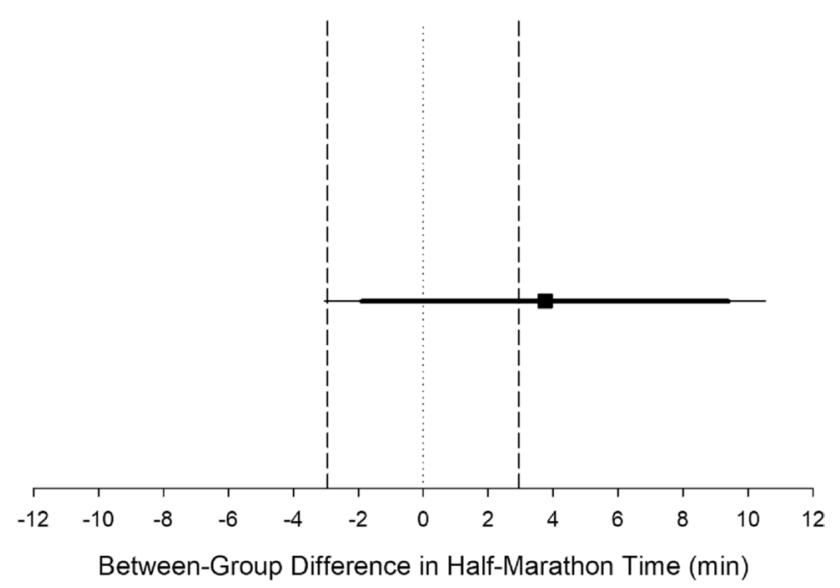

Fig. 4 Difference in half-marathon time between the mental fatigue group and the control group (black square). The thick horizontal line indicates the $90 \%$ confidence interval for such difference whereas the thin horizontal line indicates the $95 \%$ confidence interval. The dotted vertical line indicates the null hypothesis whilst the dashed vertical lines indicate the equivalence bounds in raw score feelings of mental fatigue significantly more than the control treatment (reading magazines). Therefore, the experimental treatment used in this study was successful in inducing a state of mental fatigue.

The traditional NHST analysis showed that mental fatigue did not have a significant effect on half-marathon performance. This null result is contrary to the results of most previous laboratory/indoor-based studies showing significant effects of mental fatigue on shorter-term endurance performance [2, 4, 5, 7]. One possible explanation for this null result may be the difference in fitness level between our participants and those recruited in earlier studies. Indeed, the negative effect of mental fatigue on short-term endurance performance seems to be larger in less fit subjects [12]. However, in the present study, participants' fitness level was similar or even slightly lower compared to previous experiments, hence this factor is very unlikely to explain our null result. Another potential reason might be the duration of our test of endurance performance (a real half-marathon) which is considerably longer than the time trials and time to exhaustion tests used in previous studies. However, it is generally assumed that psychological factors like mental fatigue become more, not less, influential as the duration of the endurance event increases [41, 42]. Therefore, differences in testing duration do not seem a plausible explanation for the discrepancy between our null result and the results of most previous studies. A further aspect that could be considered is the duration of the cognitive task (50 $\mathrm{min}$ ) used as experimental treatment which perhaps was not long enough to induce a state of mental fatigue. However, this speculation is in contrast with the results of our manipulation checks and with the findings of a recent meta-analysis which revealed that cognitive tasks of shorter as well as longer duration can induce mental fatigue [43]. A more plausible explanation for the discrepancy between our null result and the results of most previous studies is the difference in motivational climate. To the best of our knowledge, our study is the first experiment investigating the effects of mental fatigue during a real mass-start endurance competition in which participants competed against other participants. This head-to-head competition increases motivation which, in turn, may have reduced the negative effect of mental fatigue on endurance performance compared to previous studies in which participants were tested alone [44, 45].

In our opinion, the most likely explanation for the null results of our between-subject, posttest-only experimental study is the lack of statistical power compared to previous experimental studies that had a more powerful withinsubject design or a pretest [43]. Participants in the mental fatigue group completed the race, on average, four minutes slower than the participants in the control group with an effect size of 0.333 (Cohen's $d$ ). This effect size is similar to the effect sizes found in previous within-subject, lab-based 
a

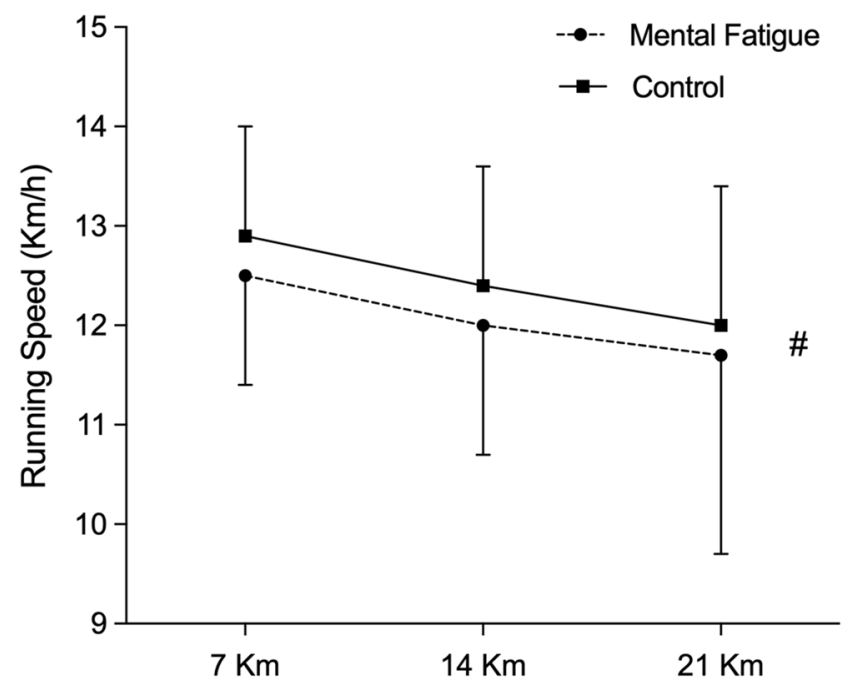

c

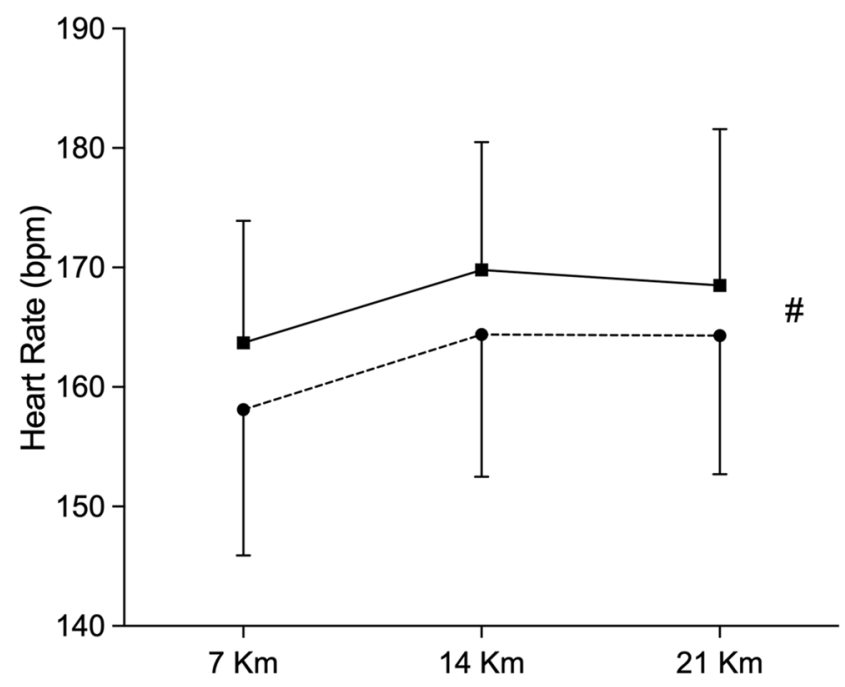

b

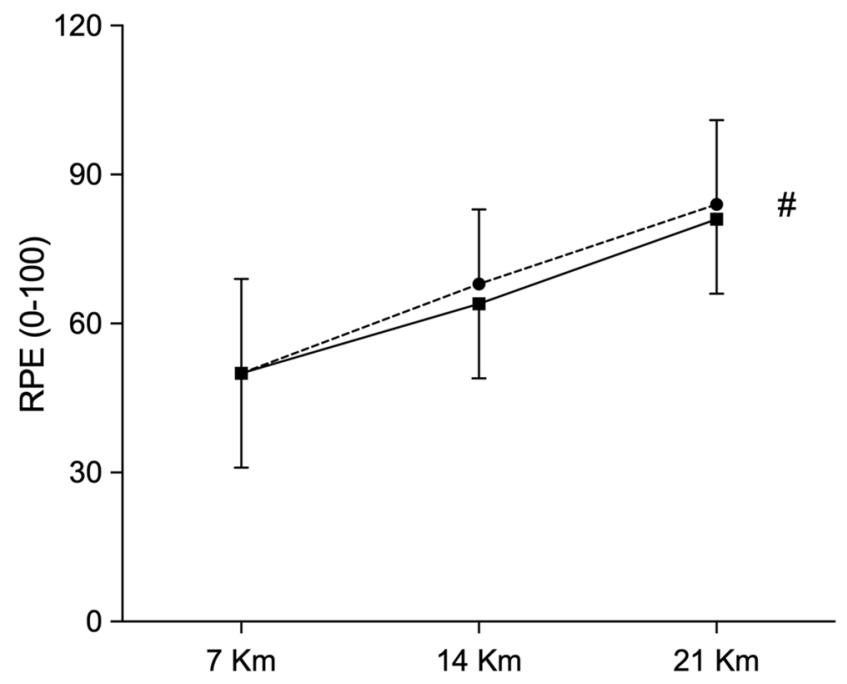

d

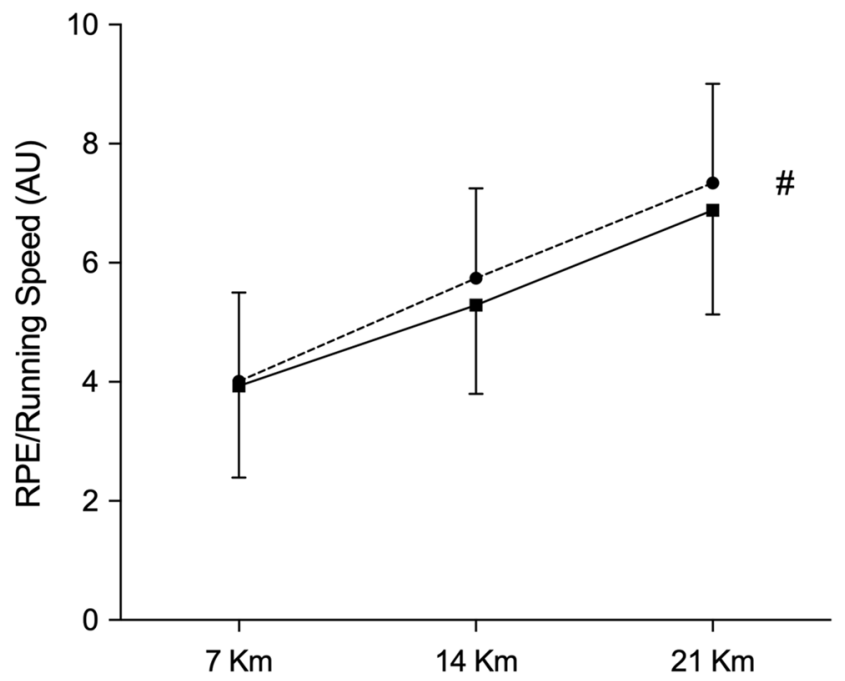

Fig. 5 Running Speed (a), RPE (b), Heart Rate (c) and RPE/Running Speed (d) during the half-marathon race in the mental fatigue group and the control group. $A U$, Arbitrary Units. Data are presented as mean \pm SD. \#Significant main effect of distance $(p<0.05)$

experiments in which the effect of mental fatigue on endurance performance was statistically significant $[5,7]$. Furthermore, the effect size in our pragmatic trial is larger than the significant pooled effect size (Hedge's $g=0.26$ ) found for the effect of prior cognitive exertion on aerobic performance in a recent meta-analysis of lab-based studies [43]. However, because of the between-group, posttest-only experimental design of our pragmatic trial, a post hoc power analysis estimated that a sample size of 286 runners ( 143 per group) would be necessary to demonstrate statistical significance for a Cohen's $d=0.333$, alpha $=5 \%$ and beta $=20 \%$. It is clear that conducting experiments of such sample size during real endurance competitions is extremely difficult. Due to the limited funding available and the logistical barriers to recruitment and testing, the maximum number of participants we were able to recruit over three consecutive years was 48. After accounting for the two dropouts during the race in the mental fatigue group, post hoc power analysis gave us a statistical power of $19.7 \%$ which is very low. Therefore, it is not surprising that the effect of mental fatigue on half-marathon performance is not significant according to NHST. However, finding a $p$ value larger than 0.05 or a $95 \%$ confidence interval including zero does not necessarily 
mean that there is 'no difference', 'no effect' or 'no association' [38].

To prevent the common misinterpretation of $p$ values larger than the alpha level as support for the absence of a true effect $[39,40]$, we also implemented the TOST equivalence testing procedure $[39,40]$. This novel statistical approach has been proposed as a more valid alternative to the magnitude based inference approach, previously used in sport performance research and recently abandoned by statisticians and scientific journals $[36,37]$. The TOST equivalence testing procedure rejected the hypothesis that mental fatigue has a beneficial effect on half-marathon performance in amateur runners but failed to reject the hypothesis that it has either a trivial or a harmful effect (Fig. 4). In other words, the results of the present study demonstrate that mental fatigue can either have no meaningful effect or a detrimental effect on half-marathon performance in amateur runners.

\section{Potential mechanisms}

Notwithstanding the limitations with regards to NHST analysis and lack of adequate power in our pragmatic trial, it is worth briefly discussing the effects of mental fatigue on other variables as they may provide some hints to the mechanisms underlying the potential negative effect of mental fatigue on long-term endurance performance.

Very small effect sizes and no statistically significant differences were found for success motivation, intrinsic motivation, and performance expectations. Therefore, it is unlikely that the potential negative effect of mental fatigue on halfmarathon performance in amateur runners was mediated by a large reduction in motivation or a nocebo effect caused by the experimental treatment [46].

In agreement with previous studies [47-49], the present experiment showed that running speed significantly decreased throughout the half-marathon in both groups (i.e. positive pacing pattern, which consists in a fast start followed by a progressive reduction in running speed). Even though no statistically significant differences were found between the two groups, running speed in the mental fatigue group was on average 3\% slower than in the control group. Heart rate values were also $3 \%$ lower throughout the entire race in the mental fatigue group compared to the control group. Moreover, RPE and the ratio between RPE and running speed during the race were similar (at $7 \mathrm{~km}$ ) or higher (at 14 and $21 \mathrm{~km}$ ) in the mental fatigue group compared to the control group. The directions of these between-group differences, although not statistically significant, are consistent with the significant differences observed in more powerful within-subject studies showing that mental fatigue [8] and the fatigue that accumulates during multi-day races [50] are associated with an increase in RPE for a given speed/ power or HR. According to the psychobiological model of self-paced endurance performance [51,52], such increase in perception of effort would lead to the conscious decision to reduce the running speed during the half-marathon race to avoid premature exhaustion with the consequent reduction in $\mathrm{HR}$ and the increase in half-marathon time. The directions of the changes in running speed, RPE, HR and half-marathon time observed in the present study are consistent with such theoretical prediction and the results of previous studies on the effects of mental fatigue on self-paced endurance performance tests in the laboratory or on an indoor track [3-5].

\section{Practical applications and directions for future research}

In conclusion, because of low statistical power, the data collected do not provide reliable evidence that mental fatigue reduces long-term endurance performance. However, the effect size of the present study is in line with previous studies on shorter-term endurance performance [8]. Furthermore, the TOST procedure suggests that mental fatigue can either have no meaningful effect or a detrimental effect on half-marathon performance in amateur runners. Given that avoiding mentally fatiguing tasks before a race is feasible and without negative side effects, it seems prudent to recommend that endurance athletes avoid or reduce engagement in tasks that may induce mental fatigue (e.g. dealing with transport and logistics, engaging with social media, ruminating, or inhibiting emotions) before any race, including those lasting more than $45 \mathrm{~min}$. Because sleep deprivation exacerbates mental fatigue [53], endurance athletes should also implement strategies to improve their sleep before an important race [54].

Additional studies with much larger sample size or two races (baseline and follow-up) will be required to draw a firmer conclusion on the hypothesis that mental fatigue has a negative effect on endurance events longer than $45 \mathrm{~min}$, and to provide more precise estimates of the effects of mental fatigue on endurance performance during outdoor mass-start competitions. Given some evidence that elite endurance athletes may be more resistant to mental fatigue than amateurs [12], future studies should include participants of higher competitive level. Further research is also required to confirm our hypothesis that the main mediator of the negative effect of mental fatigue on long-term endurance performance is primarily the increase in perception of effort associated with mental fatigue.

Acknowledgements The authors would like to thank GlaxoSmithKline (GSK) Consumer Healthcare for their monetary support and Axon Sports for providing the cognitive tasks software. The authors would also like to thank Mr Guillaume Toffoli for his support in the TOST data analysis, and Mr Antonis Kesisoglou and Mr Borja Martinez Gonzalez for their help during the data collection. 
Funding Open access funding provided by Alma Mater Studiorum Università di Bologna within the CRUI-CARE Agreement. The present study was supported by the GSK Human Performance Lab.

Availability of data and materials The data sets generated during and/or analysed during the current study are available from Dr Chiara Gattoni on reasonable request.

\section{Declarations}

Conflict of interest One of the co-authors (BVO) was previously employed by GSK which also provided financial support to Dr Chiara Gattoni during her $\mathrm{PhD}$, including the current study.

Ethical approval All procedures performed in this study were in accordance with the ethical standards of the institutional and/or national research committee (Ethics Committee of the University of Verona) and with the 1964 Helsinki declaration and its later amendments or comparable ethical standards.

Informed consent Informed consent was obtained from all individual participants included in the study.

Open Access This article is licensed under a Creative Commons Attribution 4.0 International License, which permits use, sharing, adaptation, distribution and reproduction in any medium or format, as long as you give appropriate credit to the original author(s) and the source, provide a link to the Creative Commons licence, and indicate if changes were made. The images or other third party material in this article are included in the article's Creative Commons licence, unless indicated otherwise in a credit line to the material. If material is not included in the article's Creative Commons licence and your intended use is not permitted by statutory regulation or exceeds the permitted use, you will need to obtain permission directly from the copyright holder. To view a copy of this licence, visit http://creativecommons.org/licenses/by/4.0/.

\section{References}

1. Boksem MAS, Tops M (2008) Mental fatigue: costs and benefits. Brain Res Rev 59:125-139

2. Pageaux B, Marcora SM, Lepers R (2013) Prolonged mental exertion does not alter neuromuscular function of the knee extensors. Med Sci Sports Exerc 45:2254-2264

3. Brownsberger J, Edwards A, Crowther R, Cottrell D (2013) Impact of mental fatigue on self-paced exercise. Int J Sports Med 34:1029-1036

4. MacMahon C, Schücker L, Hagemann N, Strauss B (2014) Cognitive fatigue effects on physical performance during running. $\mathrm{J}$ Sport Exerc Psychol 36:375-381

5. Pageaux B, Lepers R, Dietz KC, Marcora SM (2014) Response inhibition impairs subsequent self-paced endurance performance. Eur J Appl Physiol 114:1095-1105

6. Martin K, Thompson KG, Keegan R et al (2015) Mental fatigue does not affect maximal anaerobic exercise performance. Eur J Appl Physiol 115:715-725

7. Marcora SM, Staiano W, Manning V (2009) Mental fatigue impairs physical performance in humans. J Appl Physiol 106:857-864

8. Van Cutsem J, Marcora S, De Pauw K et al (2017) The effects of mental fatigue on physical performance: a systematic review. Sports Med 47:1569-1588
9. Van Cutsem J, De Pauw K, Buyse L et al (2017) Effects of mental fatigue on endurance performance in the heat. Med Sci Sports Exerc 49:1677-1687

10. Otani H, Kaya M, Tamaki A, Watson P (2017) Separate and combined effects of exposure to heat stress and mental fatigue on endurance exercise capacity in the heat. Eur J Appl Physiol 117:119-129

11. Pageaux B, Lepers R (2016) Fatigue induced by physical and mental exertion increases perception of effort and impairs subsequent endurance performance. Front Physiol 7:587

12. Martin K, Staiano W, Menaspà $P$ et al (2016) Superior inhibitory control and resistance to mental fatigue in professional road cyclists. PLoS ONE 11:e0159907

13. Utter AC, Kang J, Robertson RJ et al (2002) Effect of carbohydrate ingestion on ratings of perceived exertion during a marathon. Med Sci Sports Exerc 34:1779-1784

14. McCormick A, Meijen C, Marcora S (2018) Effects of a motivational self-talk intervention for endurance athletes completing an ultramarathon. Sport Psychol 32:42-50

15. Rowlands DS, Houltham SD (2017) Multiple-transportable carbohydrate effect on long-distance triathlon performance. Med Sci Sports Exerc 49:1734-1744

16. Pugh JN, Sparks AS, Doran DA et al (2019) Four weeks of probiotic supplementation reduces GI symptoms during a marathon race. Eur J Appl Physiol 119:1491-1501

17. Hansen EA, Emanuelsen A, Gertsen RM, Sørensen SSR (2014) Improved marathon performance by in-race nutritional strategy intervention. Int J Sport Nutr Exerc Metab 24:645-655

18. Del Coso J, González-Millán C, Salinero JJ et al (2016) Effects of oral salt supplementation on physical performance during a half-ironman: A randomized controlled trial. Scand J Med Sci Sports 26:156-164

19. Abbott W, Brownlee TE, Naughton RJ et al (2020) Changes in perceptions of mental fatigue during a season in professional under-23 English Premier League soccer players. Res Sports Med 28:529-539

20. Russell S, Jenkins D, Rynne S et al (2019) What is mental fatigue in elite sport? Perceptions from athletes and staff. EJSS 19:1367-1376

21. Russell S, Jenkins DG, Halson SL, et al (2021) How do elite female team sport athletes experience mental fatigue? Comparison between international competition, training and preparation camps. EJSS 1-11

22. Thompson CJ, Noon M, Towlson C et al (2020) Understanding the presence of mental fatigue in English academy soccer players. J Sports Sci 38:1524-1530

23. Fortes LS, Lima-Junior D, Nascimento-Júnior JRA et al (2019) Effect of exposure time to smartphone apps on passing decisionmaking in male soccer athletes. Psychol Sport Exerc 44:35-41

24. van Rensburg DCJ, van Rensburg AJ, Fowler P et al (2020) How to manage travel fatigue and jet lag in athletes? A systematic review of interventions. Br J Sports Med 54:960-968

25. Brick NE, MacIntyre TE, Campbell MJ (2016) Thinking and action: a cognitive perspective on self-regulation during endurance performance. Front Physiol 7:159

26. Ross RM, Jackson AS (1991) Exercise concepts, calculations, and computer applications. Med Sci Sports Exerc 23:887

27. Jackson AS, Blair SN, Mahar MT et al (1990) Prediction of functional aerobic capacity without exercise testing. Med Sci Sports Exerc 22:863

28. Borg G (1998) Borg's Perceived Exertion and Pain Scales. Human Kinetics 1

29. Tucker MA, Ganio MS, Adams JD et al (2015) Hydration status over 24-h is not affected by ingested beverage composition. J Am Coll Nutr 34:318-327 
30. Kirsch IS, Guthrie JT (1984) Adult reading practices for work and leisure. Adult Educ Q 34:213-232

31. Hart SG, Staveland LE (1988) Development of NASA-TLX (Task Load Index): Results of empirical and theoretical research. Adv Psychol 139-183

32. Matthews G, Campbell SE, Falconer S (2001) Assessment of motivational states in performance environments. Proceedings Human Factors Ergonomics Soc Annual Meeting 45:906-910

33. Hopkins WG, Marshall SW, Batterham AM, Hanin J (2009) Progressive statistics for studies in sports medicine and exercise science. Med Sci Sports Exerc 41:3-13

34. Sainani KL (2018) The Problem with" Magnitude-based Inference.” Med Sci Sports Exerc 50:2166-2176

35. Instructions for Authors Medicine \& Science in Sports \& Exercise. http://edmgr.ovid.com/msse/accounts/ifauth.htm. Accessed 2 May 2021

36. Harrison AJ, McErlain-Naylor SA, Bradshaw EJ et al (2020) Recommendations for statistical analysis involving null hypothesis significance testing. Sports Biomech 19:561-568

37. Aisbett J, Lakens D, Sainani K (2020) Magnitude Based Inference in Relation to One-sided Hypotheses Testing Procedures

38. Amrhein V, Greenland S, McShane B (2019) Scientists rise up against statistical significance. Nature 567:305-307

39. Lakens D (2017) Equivalence tests: a practical primer for $t$ tests, correlations, and meta-analyses. Soc Psychol Personal Sci $8: 355-362$

40. Lakens D, Scheel AM, Isager PM (2018) Equivalence testing for psychological research: a tutorial. Adv Methods Pract Psychol Sci 1:259-269

41. Burnley M, Jones AM (2007) Oxygen uptake kinetics as a determinant of sports performance. EJSS 7:63-79

42. Meijen C (2019) Endurance Performance in Sport: Psychological Theory and Interventions. Routledge

43. Brown DMY, Graham JD, Innes KI et al (2020) Effects of prior cognitive exertion on physical performance: a systematic review and meta-analysis. Sports Med 50:497-529
44. Brown D, Bray S (2016) Show me the money! Incentives attenuate effects of cognitive control exertion (mental fatigue) on physical endurance performance. J Exercise Movement Sport 48:149

45. Wilmore JH (1968) Influence of motivation on physical work capacity and performance. J Appl Physiol 24:459-463

46. Boot WR, Simons DJ, Stothart C, Stutts C (2013) The pervasive problem with placebos in psychology: why active control groups are not sufficient to rule out placebo effects. Perspect Psychol Sci $8: 445-454$

47. Nikolaidis PT, Ćuk I, Knechtle B (2019) Pacing of Women and Men in Half-Marathon and Marathon Races. Medicina. https:// doi.org/10.3390/medicina55010014

48. Cuk I, Nikolaidis PT, Knechtle B (2020) Sex differences in pacing during half-marathon and marathon race. Res Sports Med 28:111-120

49. Cuk I, Nikolaidis PT, Markovic S, Knechtle B (2019) Age differences in pacing in endurance running: comparison between marathon and half-marathon men and women. Medicina 55:479

50. Sanders D, Heijboer M, Hesselink MKC et al (2018) Analysing a cycling grand tour: Can we monitor fatigue with intensity or load ratios? J Sports Sci 36:1385-1391

51. Marcora S (2010) Counterpoint: afferent feedback from fatigued locomotor muscles is not an important determinant of endurance exercise performance. J Appl Physiol 108:454-456

52. Pageaux B (2014) The psychobiological model of endurance performance: an effort-based decision-making theory to explain self-paced endurance performance. Sports Med 44:1319-1320

53. Wesensten NJ, Belenky G, Thorne DR et al (2004) Modafinil vs. caffeine: effects on fatigue during sleep deprivation. Aviat Space Environ Med 75:520-525

54. Halson SL, Juliff LE (2017) Sleep, sport, and the brain. Prog Brain Res 234:13-31

Publisher's Note Springer Nature remains neutral with regard to jurisdictional claims in published maps and institutional affiliations. 